\title{
Familial Associations Between Prostate Cancer and Other Cancers
}

\section{Frank, Christoph}

2017-02-01

Frank , C , Sundquist , J , Hemminki , A \& Hemminki , K 2017 , ' Familial Associations

Between Prostate Cancer and Other Cancers ' , European Urology , vol. 71 , no. 2 , pp.

162-165 . https://doi.org/10.1016/j.eururo.2016.07.031

http://hdl.handle.net/10138/231664

https://doi.org/10.1016/j.eururo.2016.07.031

publishedVersion

Downloaded from Helda, University of Helsinki institutional repository.

This is an electronic reprint of the original article.

This reprint may differ from the original in pagination and typographic detail.

Please cite the original version. 


\title{
Familial Associations Between Prostate Cancer and Other Cancers
}

\author{
Christoph Frank $^{a}$, Jan Sundquist ${ }^{b, c}$, Akseli Hemminki ${ }^{d, e}$, Kari Hemminki $^{a, b, *}$ \\ ${ }^{a}$ Division of Molecular Genetic Epidemiology, German Cancer Research Center (DKFZ), Heidelberg, Germany; ${ }^{\mathrm{b}}$ Center for Primary Health Care Research, Lund \\ University, Malmö, Sweden; ' Stanford Prevention Research Center, Stanford University School of Medicine, Stanford, CA, USA; ${ }^{\mathrm{d} C a n c e r ~ G e n e ~ T h e r a p y ~ G r o u p, ~}$ \\ Faculty of Medicine, University of Helsinki, Helsinki, Finland; ${ }^{\mathrm{e}}$ Helsinki University Hospital Comprehensive Cancer Center, Helsinki, Finland
}

\section{Article info}

Article history:

Accepted July 20, 2016

Associate Editor:

James Catto

Keywords:

Familial cancer

Discordant cancer

Familial risk

Genetic association

\begin{abstract}
Prostate cancer (PCa) has a large familial component, but understanding of its genetic basis is fragmentary. Breast cancers may be associated with PCa, but whether this is true for other tumor types is poorly established. We used a novel approach to study familial associations of any type of cancer with PCa. We assessed the relative risk (RR) for all types of tumors as a function of the number of first-degree relatives diagnosed with PCa. We hypothesized that for a familial association to be real, the RR for a given type of cancer should increase with the number of PCa diagnoses. In families with multiple PCa patients, significantly increased risks were observed for female breast cancer (RR 1.37 for families with three men with PCa), kidney cancer (RR 2.32), nervous system tumors (RR 1.77; RR 2.40 when PCa was diagnosed before age 70 yr), and myeloma (RR 2.44; RR 6.29 when PCa was diagnosed before age $70 \mathrm{yr}$ ). Some evidence of association was also found for melanoma (RR 1.82) and endocrine tumors (RR 2.18). The consistency and magnitude of the effects suggest that familial PCa is genetically associated with breast, kidney, and nervous system tumors and myeloma. This suggestion has implications for clinical counseling and design of genetic studies.

Patient summary: It is known that prostate cancer runs in families, but it is not known whether other cancers are common in such families. We showed that at least breast, kidney, and nervous system tumors and myeloma occur more often than by chance.

(C) 2016 European Association of Urology. Published by Elsevier B.V. All rights reserved.

* Corresponding author. German Cancer Research Center (DKFZ), Im Neuenheimer Feld 580, 69120 Heidelberg, Germany. Tel. +49 6221 421800; Fax: +496221 421810.

E-mail address: k.hemminki@dkfz.de (K. Hemminki).
\end{abstract}

Prostate cancer (PCa) has the largest familial component of all common cancers: $22 \%$ of diagnosed men have a firstdegree relative-a father, a brother, or both-diagnosed with the same cancer, and high-risk families with at least three affected first-degree relatives account for $15 \%$ of familial PCa and 3\% of all PCa [1]. Nevertheless, the search for highrisk predisposing genes has not been successful. Although BRCA2 and HOXB13 mutations confer an increased individual risk of PCa, their population impact is modest [2]. In addition, numerous low-penetrance loci have been described for PCa, and the known loci and the above mutations combined are estimated to account for about one-third of familial risk of PCa [2]. Families with BRCA2 mutations would be expected to manifest other BRCA2-related cancers, but at the population level, only breast cancer shows familial associations with PCa [3,4]. In the present study, we applied a novel approach to search for familial associations between of PCa and other cancers by assessing clustering of all types of cancers in families with increasing numbers of PCa. The large population size of the Swedish Family-Cancer Database allows a powerful search of discordant cancers, even in families with three or more patients with PCa. The 
data have implications for familial genetic counseling and for identifying novel predisposing genes for PCa.

In the Swedish Family-Cancer Database, 15.7 million persons are organized in families with cancer data from the Swedish Cancer Registry. The offspring generation (8.5 million persons with 427196 cancers) comprised people born from 1932 onward. The latest follow-up of the database includes data through 2012; therefore, the maximum age of offspring was $80 \mathrm{yr}$, whereas the ages of their biological parents (the parental generation) were not limited. Using the seventh revision of the International Classification of Diseases, 36 different cancers were analyzed, but data on only 23 were reported because familial cases were few among 13 cancers. The follow-up for cancer in the offspring generation started from the beginning of 1958, the birth year, or the immigration year, whichever came latest. The follow-up was terminated when a person was diagnosed with cancer, emigrated, or died or at the end of 2012, whichever came first. Analysis by TNM category was hampered because the classification was introduced in 2002.

Methods of calculating familial relative risks (RRs) for offspring cancer when first-degree relatives (parents and/or siblings) were diagnosed with cancer are described elsewhere [1]. Briefly, incidence rates for persons with affected relatives were compared with rates for those whose relatives had no cancer. Incidence rates were obtained by counting cases and person-years according to family history, stratified for sex, age group, calendar period, residential area, and socioeconomic status to account for potential confounders. These variables were used as covariates for model building for adjusted RRs, corresponding 95\% and 99\% confidence intervals (CIs), and a trend test.

Figure 1 shows familial risks for cancers other than PCa as a function of the number of men diagnosed with PCa; the comparison was to families with no PCa (RR 1.00). Data are shown for six cancers with significantly increased RRs in families with at least three PCa patients. Data on all 23 cancers, including PCa, are shown in Supplementary Table 1. For all six cancers in Figure 1, RRs increased systematically by the number of affected family members with PCa. In families with at least three PCa cases, increased risks of myeloma (RR 2.44; 95\% CI, 1.24-4.82), kidney cancer (RR 2.32; 95\% CI, 1.23-4.36), and nonthyroid endocrine tumors (RR 2.18; 95\% CI, 1.06-4.49) were noted. Increased risks were also observed for melanoma (RR 1.82; $95 \% \mathrm{CI}, 1.18-2.80$ ), nervous system tumors (RR 1.77; 95\% CI, 1.08-2.91), and female breast cancer (RR 1.37; 95\% CI, $1.02-$ 1.86). RRs for male breast cancer were not significant. For comparison, Supplementary Table 1 shows that the risk of PCa was increased almost eightfold in families in which three members already had PCa.

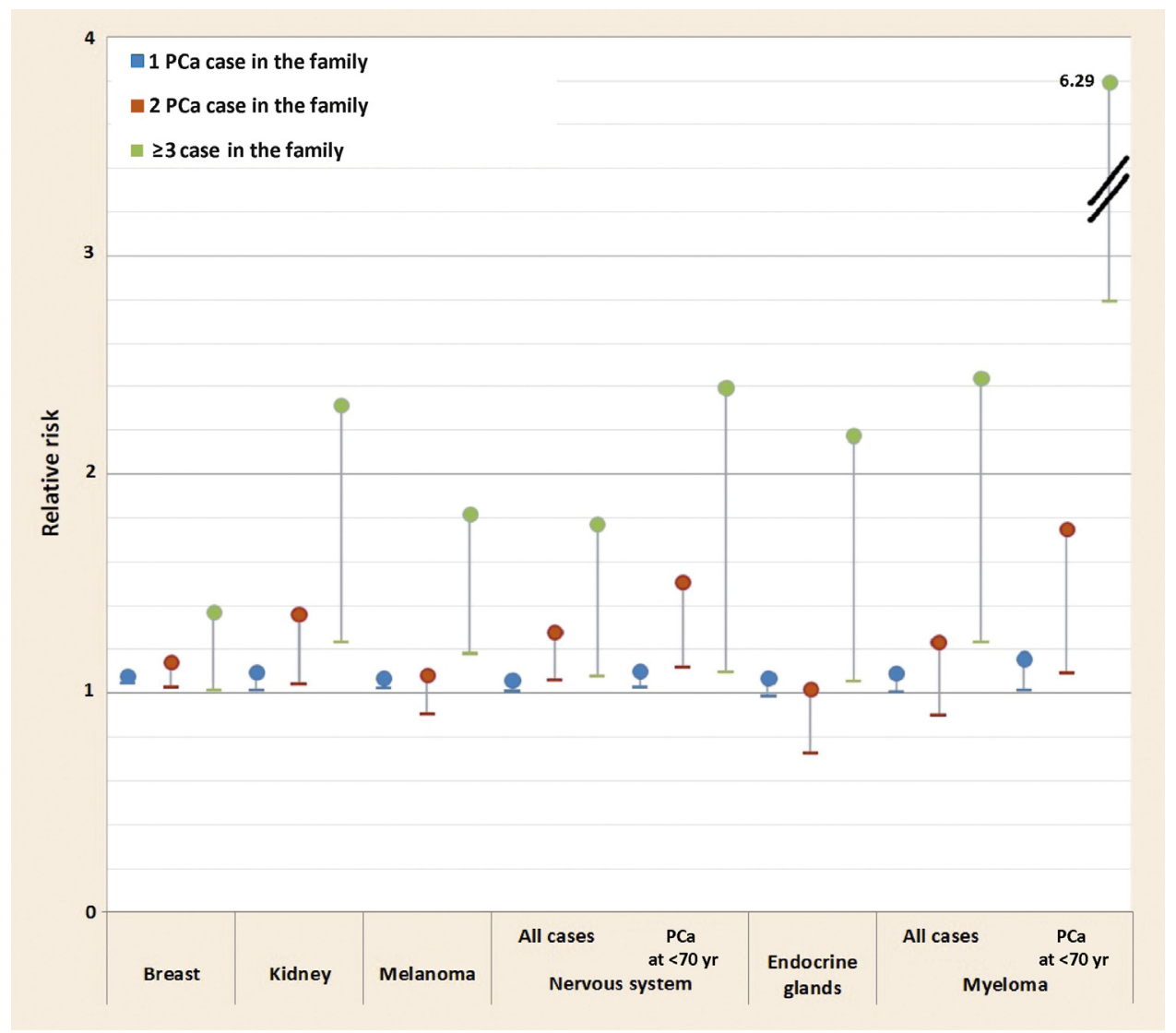

Fig. 1 - Relative risks of six cancers associated with prostate cancer (PCa) in families of increasing numbers of men diagnosed with PCa (one, two, and three members with PCa in one family, shown by different colors). The comparison is to families without PCa. The downward bars show the lower bounds of the $95 \%$ confidence intervals. The full data can be found in Supplementary Table 1.

$\mathrm{PCa}=$ prostate cancer. 
Table 1 - Risks of prostate cancer when family members were diagnosed with discordant cancers

\begin{tabular}{|c|c|c|c|c|c|c|c|}
\hline \multirow[t]{2}{*}{ Cancer site in the family } & \multirow[t]{2}{*}{ Cases with negative family history } & \multicolumn{3}{|c|}{1 cancer case in the family } & \multicolumn{3}{|c|}{$\geq 2$ cancer cases in the family } \\
\hline & & Cases & $\mathrm{RR}$ & $95 \% \mathrm{CI}$ & Cases & $\mathrm{RR}$ & $95 \% \mathrm{CI}$ \\
\hline Upper aerodigestive tract & 60798 & 1061 & 0.98 & $0.91-1.05$ & 5 & 0.50 & $0.18-1.41$ \\
\hline Stomach & 59793 & 2041 & 0.97 & $0.93-1.02$ & 30 & 0.97 & $0.65-1.45$ \\
\hline Colorectum & 56147 & 5451 & 1.02 & $0.98-1.05$ & 266 & 0.99 & $0.85-1.15$ \\
\hline Liver & 60471 & 1379 & 1.01 & $0.95-1.08$ & 14 & 0.95 & $0.50-1.82$ \\
\hline Pancreas & 60494 & 1352 & 0.98 & $0.92-1.04$ & 18 & 1.01 & $0.59-1.71$ \\
\hline Lung & 58584 & 3188 & 0.95 & $0.91-0.99$ & 92 & 0.76 & $0.59-0.96$ \\
\hline Breast & 54966 & 6523 & 1.12 & $1.08-1.16$ & 375 & 1.08 & $0.95-1.24$ \\
\hline Cervix & 61036 & 824 & 0.93 & $0.86-1.02$ & 4 & 0.90 & $0.26-3.14$ \\
\hline Endometrium & 60361 & 1489 & 1.08 & $1.01-1.14$ & 14 & 0.70 & $0.38-1.30$ \\
\hline Ovary & 60606 & 1240 & 1.06 & $0.99-1.13$ & 18 & 1.28 & $0.75-2.19$ \\
\hline Prostate & 46668 & 12985 & 2.20 & $2.14-2.27$ & 2211 & 4.81 & $4.51-5.14$ \\
\hline Testis & 61721 & 141 & 1.09 & $0.90-1.33$ & 2 & 2.59 & $0.50-13.48$ \\
\hline Kidney & 60294 & 1544 & 1.05 & $0.99-1.12$ & 26 & 1.44 & $0.92-2.26$ \\
\hline Urinary bladder & 59562 & 2262 & 1.00 & $0.95-1.04$ & 40 & 0.82 & $0.58-1.16$ \\
\hline Melanoma & 60300 & 1527 & 1.06 & $1.00-1.12$ & 37 & 1.03 & $0.72-1.47$ \\
\hline Skin, squamous cell & 59908 & 1913 & 0.93 & $0.88-0.99$ & 43 & 1.06 & $0.73-1.53$ \\
\hline Nervous system & 60323 & 1507 & 1.08 & $1.01-1.14$ & 34 & 1.66 & $1.11-2.48$ \\
\hline Thyroid gland & 61431 & 428 & 1.13 & $1.00-1.27$ & 5 & 2.08 & $0.67-6.41$ \\
\hline Endocrine glands & 60939 & 918 & 1.11 & $1.04-1.20$ & 7 & 0.86 & $0.38-1.95$ \\
\hline Non-Hodgkin lymphoma & 60380 & 1464 & 1.00 & $0.94-1.06$ & 20 & 0.91 & $0.55-1.52$ \\
\hline Myeloma & 61073 & 784 & 1.06 & $0.98-1.15$ & 7 & 1.07 & $0.45-2.59$ \\
\hline Leukemia & 60366 & 1476 & 1.07 & $1.00-1.15$ & 22 & 1.20 & $0.68-2.11$ \\
\hline CUP & 60145 & 1700 & 1.05 & $0.98-1.11$ & 19 & 1.12 & $0.63-1.97$ \\
\hline
\end{tabular}

We carried out a separate analysis in families in which all PCa cases in any relatives were diagnosed before age $70 \mathrm{yr}$. The risk of nervous system cancer and myeloma were further increased in these families, reaching RRs of 2.40 (95\% CI, 1.10-5.23) and 6.26 (95\% CI, 2.79-14.17), respectively, in families with at least three members with PCa (Fig. 1 and Supplementary Table 1).

We studied with some detail the associated endocrine organs and histology of the associated breast, kidney, and nervous system tumors but found no obvious differences in the background distributions.

In the reverse analysis, the risk of PCa was assessed in families of discordant cancers (Table 1). Because familial clustering for other cancers is rarer than for PCa, only families with one or at least two non-PCa cancers could be considered. The risk of PCa was increased systematically only in families with nervous system tumors, reaching an RR of 1.66 (95\% CI, 1.11-2.48) in families presenting with at least two nervous system cancers. PCa was increased in families with a single member diagnosed with breast cancer (RR 1.12; 95\% CI, 1.08-1.16) and endometrial cancer (RR 1.08; 95\% CI, 1.01-1.14), melanoma (RR 1.06; 95\% CI, 1.00-1.12), and endocrine gland tumors (RR 1.11;95\% CI, 1.04-1.20).

Familial clustering may be due to shared genes, shared environment, their interactions, or increased screening [5]. Strong environmental risk factors linking PCa with endocrine and nervous system tumors, myeloma, or melanoma are not apparent, and thus a genetic contribution is likely [2]. For the association with breast cancer, BRCA2 mutations may contribute; however, because the PCa association was stronger in families with a single breast cancer in comparison to those with multiple breast cancers, shared hormonal pathways may be the main common mechanism [6,7]. The RR of PCa in Swedish BRCA2 mutation carriers was reported to be only 2.3 [8]. In previous studies investigating familial risks between pairs of cancers, weak associations of PCa with breast cancer and myeloma were reported [3,9]. Family studies from Iceland detected associations of kidney cancer and melanoma with PCa [10]. In the Utah population database, PCa risk was found in melanoma families [11]. The kind of genes that could explain the observed associations is open to speculation. Individual low-risk genes contribute minimally to familial risk, and it is not known whether a polygenic set of low-risk PCa-related alleles would cause risk for another cancer $[1,2,12]$. Similarly, high-risk genes shared by PCa and other cancers are not known. By exclusion, the remaining options are as yet unidentified moderate-risk genes and/or geneenvironment interactions.

Multiple testing may be an issue in exploratory studies such as the present one; however, the results are unlikely to be due to chance when they are supported by "dose response," namely, significant and increasing RRs for a defined cancer in families with increasing numbers of patients with PCa [3]. If two RRs are significant at a minimal probability of 0.05 (ie, melanoma in Supplementary Table 1), the combined probability is $0.05 \times 0.05=0.0025$. Incidentally, this is the adjusted significance using the Bonferroni correction with 22 cancers tested [3]. For breast cancer, three RRs were significant at $p<0.01$, yielding a combined probability of $<10^{-6}$.

In summary, the convincing dose-response data showing increased risks of kidney and nervous system tumors and 
myeloma provide strong familial evidence that they share as yet unknown predisposing genes with PCa. The evidence for melanoma and endocrine tumors is less compelling, and for breast cancer, the role of BRCA2 may not be sufficient to explain the associations.

Author contributions: Kari Hemminki had full access to all the data in the study and takes responsibility for the integrity of the data and the accuracy of the data analysis.

Study concept and design: K. Hemminki.

Acquisition of data: Sundquist.

Analysis and interpretation of data: Frank, K. Hemminki, A. Hemminki.

Drafting of the manuscript: K. Hemminki, A. Hemminki.

Critical revision of the manuscript for important intellectual content: A.

Hemminki, Sundquist, Frank.

Statistical analysis: Frank.

Obtaining funding: K. Hemminki, Sundquist, A. Hemminki.

Administrative, technical, or material support: K. Hemminki.

Supervision: K. Hemminki.

Other (specify): None.

Financial disclosures: Kari Hemminki certifies that all conflicts of interest, including specific financial interests and relationships and affiliations relevant to the subject matter or materials discussed in the manuscript (eg, employment/ affiliation, grants or funding, consultancies, honoraria, stock ownership or options, expert testimony, royalties, or patents filed, received, or pending), are the following: Akseli Hemminki is a shareholder of Targovax ASA and an employee and shareholder of TILT Biotherapeutics Ltd.

Funding/Support and role of the sponsor: This work was funded by the German Cancer Aid, the EU Transcan funding by the German Federal Ministry of Education and Research, University of Helsinki, Helsinki University Central Hospital, and the Swedish Research Council. The funding sources were not involved in the study design, data collection, analysis or interpretation, the writing of the manuscript or the decision to submit the manuscript for publication.

Acknowledgment statement: Akseli Hemminki is Jane and Aatos Erkko Professor of Oncology at the University of Helsinki.

\section{Appendix A. Supplementary data}

Supplementary data associated with this article can be found, in the online version, at http://dx.doi.org/10.1016/j. eururo.2016.07.031.

\section{References}

[1] Frank C, Fallah M, Sundquist J, Hemminki A, Hemminki K. Population landscape of familial cancer. Sci Rep 2015;5:12891.

[2] Attard G, Parker C, Eeles RA, et al. Prostate cancer. Lancet 2016;387: 70-82.

[3] Hemminki K, Sundquist J, Brandt A. Do discordant cancers share familial susceptibility? Eur J Cancer 2012;48:1200-7.

[4] Rahman N. Realizing the promise of cancer predisposition genes. Nature 2014;505:302-8.

[5] Bermejo JL, Hemminki K. Familial risk of cancer shortly after diagnosis of the first familial tumor. J Natl Cancer Inst 2005;97: 1575-9.

[6] Risbridger GP, Davis ID, Birrell SN, Tilley WD. Breast and prostate cancer: more similar than different. Nat Rev Cancer 2010;10: 205-12.

[7] Hemminki K, Forsti A, Chen B. Breast and prostate cancer: familial associations. Nat Rev Cancer 2010;10:523.

[8] Johannsson O, Loman N, Moller T, Kristoffersson U, Borg A, Olsson H. Incidence of malignant tumours in relatives of BRCA1 and BRCA2 germline mutation carriers. Eur J Cancer 1999;35:1248-57.

[9] Frank C, Fallah M, Chen T, et al. Search for familial clustering of multiple myeloma with any cancer. Leukemia 2016;30:627-32.

[10] Amundadottir LT, Thorvaldsson S, Gudbjartsson DF, et al. Cancer as a complex phenotype: pattern of cancer distribution within and beyond the nuclear family. PLoS Med 2004;1:e65.

[11] Larson AA, Leachman SA, Eliason MJ, Cannon-Albright LA. Population-based assessment of non-melanoma cancer risk in relatives of cutaneous melanoma probands. J Invest Dermatol 2007;127: 183-8.

[12] Sampson JN, Wheeler WA, Yeager M, et al. Analysis of heritability and shared heritability based on genome-wide association studies for thirteen cancer types. J Natl Cancer Inst 2015;107:djv279. 